\title{
Níveis pressóricos de uma comunidade indígena do cerrado brasileiro
}

\section{Pressorial levels of an indigenous community of the Brazilian closure}

\author{
Ariadne Cristinne Pereira de Moura ${ }^{1}$, Benedita Josefina da Silva ${ }^{1}$, \\ Thalise Yuri Hattori ${ }^{2}$, Vagner Ferreira do Nascimento ${ }^{3}$, Mariano \\ Martinez Espinosa ${ }^{4}$, Marina Atanaka ${ }^{5}$, Elba Regina Sampaio \\ de Lemos $^{6}$, Ana Cláudia Pereira Terças-Trettel ${ }^{7}$
}

\section{Resumo}

\begin{abstract}
Buscou-se analisar os níveis pressóricos dos Haliti-Paresí e sua relação com alguns aspectos sociodemográficos. Trata-se de estudo transversal, descritivo e com abordagem quantitativa, realizado com a comunidade indígena Haliti-Paresí. A coleta de dados foi realizada em dezembro de 2015 com os residentes da terra indígena Utiariti. Realizou-se a análise estatística bivariada da Pressão Arterial (PA), Pressão Arterial Sistólica (PAS) e Pressão Arterial Diastólica (PAD) em função das variáveis independentes, sendo que as variáveis que apresentaram significância estatística com $\mathrm{p}<0,05$ foram utilizadas para construir o modelo de regressão de Poisson robusto. Foi possível identificar o predomínio de jovens e com ensino fundamental incompleto. A hipertensão no estágio 1 apresentou prevalência de PA $(21,85 \%)$, PAS $(14,29 \%)$ e PAD $(17,65 \%)(p<0,001)$. $\mathrm{Na}$ análise bivariada foram identificadas significâncias estatísticas para PA e PAS nas variáveis independentes sexo masculino, idade maior que 60 anos e realizar a atividade agrícola, caça, pesca e coleta. No entanto, a PAD apresentou as mesmas significâncias estatísticas supracitadas, porém sem associação com idade. Faz-se necessária a realização de ações de promoção, prevenção e elaboração de planos estratégicos que visam ampliar os hábitos saudáveis e consequente elevação da qualidade de vida desses indígenas.
\end{abstract}

Palavras-chave: Hipertensão. Epidemiologia. População indígena. Saúde de populações indígenas.

${ }^{1}$ Graduação em Enfermagem pela Universidade do Estado de Mato Grosso, Tangará da Serra, Mato Grosso, Brasil.

${ }^{2}$ Mestrado em Ciências da Saúde pela Universidade Federal da Grande Dourados, Dourados, Mato Grosso do Sul, Brasil. Professor Assistente do Curso de Enfermagem da Universidade do Estado de Mato Grosso, Tangará da Serra, Mato Grosso, Brasil.

${ }^{3}$ Doutorado em Bioética pelo Centro Universitário São Camilo, São Paulo, São Paulo, Brasil. Professor Adjunto do Curso de Enfermagem da Universidade do Estado de Mato Grosso, Tangará da Serra, Mato Grosso, Brasil.

${ }^{4}$ Doutorado em Ciências e Engenharia de Materiais pela Universidade de São Paulo, São Paulo, Brasil. Professor Associado III do Departamento de Estatística da Universidade Federal de Mato Grosso, Cuiabá, Mato Grosso, Brasil.

${ }^{5}$ Doutorado em Saúde Pública pela Escola Nacional de Saúde Pública, Rio de Janeiro, Rio de Janeiro, Brasil. Professora Adjunta do Instituto de Saúde Coletiva da Universidade Federal de Mato Grosso, Cuiabá, Mato Grosso, Brasil.

${ }^{6}$ Doutorado em Medicina Tropical pela Fundação Oswaldo Cruz, Rio de Janeiro, Rio de Janeiro, Brasil. Pesquisadora do Laboratório de Hantaviroses e Ricketsioses do Instituto Oswaldo Cruz, Rio de Janeiro, Rio de Janeiro, Brasil.

${ }^{7}$ Doutorado em Medicina Tropical pela Fundação Oswaldo Cruz, Rio de Janeiro, Rio de Janeiro, Brasil. Docente Adjunta do Departamento de Enfermagem da Universidade do Estado de Mato Grosso, Tangará da Serra, Mato Grosso, Brasil. E-mail: ana.claudia@unemat.br 


\begin{abstract}
The aim was to analyze the pressure levels of the Haliti-Paresí and their relationship with some sociodemographic aspects. It is a cross-sectional, descriptive study with a quantitative approach, carried out with the Haliti-Paresí indigenous community. Data collection was carried out in December 2015 with residents of Utiariti indigenous land. The bivariate statistical analysis of the arterial pressure (BP), systolic arterial pressure (SBP) and diastolic blood pressure (DBP) was performed as a function of the independent variables, and the variables that presented statistical significance with $\mathrm{p}<0.05$, were used to construct the robust Poisson regression model. It was possible to identify the predominance of young people with incomplete primary education. Hypertension in stage 1 presented a prevalence of AP (21.85\%), SBP (14.29\%) and DBP (17.65\%) $(\mathrm{p}<0.001)$. In the bivariate analysis, statistical significance was identified for PA and SBP in the independent variables male sex, age $>60$ years and to perform the agricultural activity, hunting, fishing and collection. However, PAD presented the same statistical significance as above, but without association with age. It is necessary to carry out actions of promotion, prevention and elaboration of strategic plans that aim to increase the healthy habits and consequent elevation of the quality of life of these natives.
\end{abstract}

Keywords: Hypertension. Epidemiology. Indigenous population. Health of indigenous populations.

\section{Introdução}

A hipertensão arterial sistêmica (HAS) é uma condição clínica caracterizada pela elevação dos níveis pressóricos frequentemente associada a distúrbios metabólicos e alterações funcionais e estruturais de órgãos-alvo, sendo agravada pela presença de outros fatores de risco, como dislipidemia, obesidade, intolerância à glicose e diabetes mellitus. ${ }^{(1)}$

Dados epidemiológicos no Brasil revelaram que o número de pessoas diagnosticadas com hipertensão cresceu 14,20\% em 2016. ${ }^{(2)} \mathrm{Em}$ um estudo realizado nas capitais brasileiras e Distrito Federal foi revelado que de $24,10 \%$ da população que referiu HAS, $23,60 \%$ eram do sexo feminino.(3) As diferenças regionais são importantes neste cenário; o estado do Rio de Janeiro, por exemplo, apresentou a maior prevalência de diagnóstico médico de hipertensão, e Palmas a menor, sendo estas diferenças influenciadas por inúmeros fatores. Já a prevalência da hipertensão em indígenas é reflexo da transição epidemiológica que estes passaram a vivenciar, decorrente da inserção de alimentos industrializados e ricos em açúcar, além do uso abusivo de bebidas alcoólicas. ${ }^{(4)}$
Essa transição epidemiológica, com destaque para os aspectos nutricionais, pode ser explicada pela restrição territorial que essa população foi submetida associada com a expansão das cidades. A facilidade de contato com os hábitos ocidentais fez com que deixassem de se alimentar de produtos tradicionais e naturais. ${ }^{(5)}$ Além disso, a diminuição das atividades culturais, como a caça e a pesca, e a introdução da economia monetária do trabalho assalariado resultaram em menores níveis de atividade física e em um acesso cada vez maior a alimentos processados. ${ }^{(6)}$

Um estudo internacional com comunidades indígenas também detectou o aumento da prevalência de hipertensão arterial, variando de $27 \%$ a 56\% de acordo com a idade. ${ }^{(7)}$ Estudo realizado com os índios Suruí em Rondônia ${ }^{(8)}$ verificou baixa prevalência de níveis tensionais altos quando comparado com estudos internacionais, ou seja, HAS de 2,80\% entre indivíduos com 20 anos ou mais de idade. ${ }^{(8)}$

Os povos indígenas de Mato Grosso vivenciaram o mesmo contexto descrito acima; essa população está distribuída em 65 etnias, sendo o estado com a maior diversidade cultural do País. Neste cenário, os indígenas Haliti-Paresí 
se destacam com 2.022 indivíduos, ${ }^{(9)}$ habitam a região do Médio-Norte mato-grossense, e possuem seus territórios demarcados nos municípios de Tangará da Serra, Campo Novo do Parecis, Sapezal, Diamantino, Nova Marilândia, Conquista do Oeste e Barra do Bugres. ${ }^{(10)}$

Em decorrência dessa diversidade cultural e dos poucos estudos que descrevam o perfil de saúde dos indígenas mato-grossenses, torna-se primordial a execução de pesquisas que abordem as doenças crônicas nessas comunidades, a fim de ampliar o conhecimento, possibilitando reflexões que direcionem para ações que promovam melhoria da qualidade de vida. Neste sentido, objetivou-se analisar os níveis pressóricos dos Haliti-Paresí e sua relação com alguns aspectos sociodemográficos.

\section{Material e Método}

Trata-se de estudo transversal, descritivo e com abordagem quantitativa, realizado com a comunidade indígena Haliti-Paresí.

Foi realizado nas aldeias Haliti-Paresí pertencentes ao município de Campo Novo do Parecis, no estado de Mato Grosso, Brasil, nas aldeias Seringal/Cabeça do Seringal, Chapada, 4 Cachoeiras, Bacaval, Wazare, Morrim, Utiariti, Sacre 2 e Bacaiuval. A área indígena localiza-se na Região Oeste deste município até a divisa com o município de Sapezal.

Os dados foram coletados em dezembro de 2015, pelos pesquisadores, em todo o universo populacional das aldeias Seringal/Cabeça do Seringal, Chapada, 4 Cachoeiras, Bacaval, Morrim, Utiariti, Sacre 2, Bacaiuval e Wazare. O espaço geográfico onde estão localizadas as terras dos Haliti-Paresí é o local mais desejado pelo agronegócio, pois está circundado pelas maiores regiões produtoras de grãos do estado de Mato Grosso e do Brasil. ${ }^{(9)}$

Foram incluídos todos os indígenas maiores de 18 anos, residentes nas aldeias supracitadas, que aceitaram participar do estudo e assinaram o Termo de Consentimento Livre e Esclarecido (TCLE).
A exclusão deu-se para os que não estavam na faixa etária estabelecida.

Para a coleta de dados estabeleceu-se ponto de apoio em cada aldeia, conforme indicação do cacique, que os convocou para participação no estudo. Inicialmente a equipe apresentou os objetivos e finalidades do projeto em português, considerando que em todas as aldeias o português é língua fluente; sequencialmente foi entregue o TCLE, que, após assinado, deu início à coleta de dados. A entrevista foi realizada individualmente, em local reservado e com duração de 30 minutos, em média. Posteriormente ao preenchimento do questionário, procedeu-se a verificação dos dados antropométricos e aferição dos sinais vitais.

Para a avaliação antropométrica utilizouse balança digital Filizola ${ }^{\circledR}$ com capacidade de 150 quilogramas e intervalo de 100 gramas. Para aferição da altura os indígenas foram posicionados em pé, descalços, em posição ereta, retirando-se todos os adornos contidos na cabeça; utilizou-se antropômetro Nutri-Vida.

Para a medida da pressão arterial foram seguidas as recomendações da VI Diretrizes Brasileiras de Hipertensão Arterial, ${ }^{(1)}$ e aconteceu em dois momentos distintos: no início e ao final das entrevistas que duraram cerca de 30 minutos; caso o paciente referisse ter praticado exercícios físicos, ingerido café ou bebida alcoólica ou ter fumado na última meia hora anterior à entrevista, optou-se pela verificação da PA (as duas medidas) ao final da entrevista, sempre respeitando o mínimo de um minuto entre as duas verificações. Entre os adolescentes (18-24 anos), na interpretação dos valores de PA foram considerados a idade, sexo e altura e classificados de acordo com a definição estabelecida pelo National High Blood Pressure Education Program Working Group on High Blood Pressure in Children and Adolescents.

Todos os esfigmomanômetros utilizados na coleta de dados passaram por um processo de checagem e calibração das suas funções, através da comparação com medidas feitas em aparelho com coluna de mercúrio. Assim, os indígenas foram posicionados sentados, pernas descruzadas, 
pés apoiados no chão, dorso recostado na cadeira e relaxado. O braço estava no nível do ponto médio do esterno ou quarto espaço intercostal, livre de roupas, apoiado, com a palma da mão voltada para cima e o cotovelo ligeiramente fletido. Já entre os adolescentes a diferença é que a medição foi feita no braço direito, em virtude da possibilidade de coarctação de aorta, o que pode levar a leituras falsas (baixas) no braço esquerdo, apoiado ao nível do coração.

Para a aferição obteve-se a circunferência aproximadamente no meio do braço para direcionar a escolha do manguito de tamanho adequado ao braço; posteriormente o mesmo foi posicionado no braço sem deixar folgas, 2 a 3 centímetros acima da fossa cubital e centralizado sobre a artéria braquial. Realizou-se então a estimativa do nível da pressão sistólica pela palpação do pulso radial; assim, palpou-se a artéria braquial na fossa cubital e colocou-se a campânula do estetoscópio sem compressão excessiva e começou-se a inflar rapidamente até ultrapassar 20 a $30 \mathrm{mmHg}$ o nível estimado da pressão sistólica, obtido pela palpação com posterior deflação lentamente (velocidade de $2 \mathrm{mmHg}$ por segundo) e determinação da pressão sistólica pela ausculta do primeiro som e da pressão diastólica no desaparecimento dos sons. Confirmou-se o desaparecimento dos sons mantendo ausculta por cerca de 20 a $30 \mathrm{mmHg}$ abaixo do último som. Os manguitos utilizados respeitaram o tamanho do braço de cada indivíduo avaliado.

Foram consideradas como variáveis dependentes a pressão arterial (PA), pressão arterial sistólica (PAS) e pressão arterial diastólica (PAD). Já as variáveis independentes utilizadas foram: sexo (masculino, feminino), idade em faixas (menor de 20 anos, entre 20 e 59 anos, com 60 anos ou mais), anos de escolaridade (analfabeto, ensino fundamental incompleto/ completo, ensino médio incompleto/completo, ensino superior incompleto/completo), zona de residência (aldeias 4 Cachoeiras, Bacaiuval, Bacaval, Chapada, Sacre 2, Seringal, Utiariti e Wazare), atividade que realiza na aldeia (agricultura, caça, pesca e coleta no cerrado; cuidados com a casa e com a família; estudante e brincadeiras e atividades da infância) e realiza atividades fora da aldeia (sim e não).

Os dados foram sistematizados em planilha eletrônica e analisados utilizando-se o programa Statistical Pakage for the Social Sciences (SPSS) versão 20.0, e realizada a análise bivariada da PA, PAS e PAD em função das variáveis independentes, sendo que as variáveis que apresentaram significância estatística com $p<0,05$ e IC de 95\% foram utilizadas para construir o modelo de regressão de Poisson robusto.

Este estudo integra a pesquisa matricial "Situação de Saúde dos Haliti-Paresí", que foi aprovada pelo comitê de ética em pesquisa do Hospital Universitário Júlio Muller da Universidade Federal de Mato Grosso - UFMT, tendo certificado de apresentação para apreciação ética ${ }^{\circ}$ 04647412.0.1001 e aprovação da Comissão Nacional de Ética em Pesquisa (CONEP) sob protocolo $819.939 \backslash 2014$.

\section{Resultados}

Integraram o estudo 238 indígenas residentes nas sete aldeias da terra indígena Paresí, sendo que o perfil sociodemográfico da comunidade Haliti-Paresí não apresentou diferença entre os sexos, porém predominaram jovens na faixa etária de 20 a 59 anos (média de 29,3 anos), que cursaram o ensino fundamental incompleto, maioria residentes na aldeia Bacaval $(23,53 \%)$ que realizaram em seu cotidiano as atividades de acordo com a divisão social, ou seja, entre os homens predominaram a agricultura, caça, pesca e coleta no cerrado; entre as mulheres, cuidados com a casa e com a família; para os adolescentes o estudo e as brincadeiras foram as principais atividades. Dos participantes, 19,75\% deles realizaram alguma atividade fora da aldeia, conforme Tabela 1. 
Tabela 1 - Variáveis sociodemográficas dos indígenas Haliti-Paresí, Mato Grosso, Brasil, 2016.

\begin{tabular}{|c|c|c|c|c|c|}
\hline Variáveis & Categorias & Frequência (n) & Porcentagem (\%) & IC $95 \%$ & $\mathbf{p}$ \\
\hline \multirow{2}{*}{ Sexo } & Feminino & 121 & 50,84 & $(44,30 ; 57,36)$ & \multirow{2}{*}{0,795} \\
\hline & Masculino & 117 & 49,16 & $(42,64 ; 55,70)$ & \\
\hline \multirow{3}{*}{ Faixa etária } & $<20$ anos & 65 & 27,31 & $(21,76 ; 33,44)$ & \multirow{3}{*}{$<0,001$} \\
\hline & 20-59 anos & 152 & 63,87 & $(57,41 ; 69,97)$ & \\
\hline & 60 anos ou mais & 21 & 8,82 & $(5,54 ; 13,17)$ & \\
\hline \multirow{7}{*}{ Escolaridade } & Analfabeto & 20 & 8,40 & $(5,21 ; 12,68)$ & \multirow{7}{*}{$<0,001$} \\
\hline & $\begin{array}{l}\text { Ensino fundamental } \\
\text { incompleto }\end{array}$ & 118 & 49,58 & $(43,06 ; 56,11)$ & \\
\hline & $\begin{array}{l}\text { Ensino fundamental } \\
\text { completo }\end{array}$ & 17 & 7,14 & $(4,22 ; 11,19)$ & \\
\hline & $\begin{array}{l}\text { Ensino médio } \\
\text { incompleto }\end{array}$ & 37 & 15,55 & $(11,19 ; 20,79)$ & \\
\hline & $\begin{array}{l}\text { Ensino médio } \\
\text { completo }\end{array}$ & 34 & 14,29 & $(10,10 ; 19,39)$ & \\
\hline & $\begin{array}{l}\text { Ensino superior } \\
\text { incompleto }\end{array}$ & 6 & 2,52 & $(0,93 ; 5,41)$ & \\
\hline & $\begin{array}{l}\text { Ensino superior } \\
\text { completo }\end{array}$ & 6 & 2,52 & $(0,93 ; 5,41)$ & \\
\hline \multirow{8}{*}{$\begin{array}{l}\text { Local de } \\
\text { residência }\end{array}$} & Aldeia 4 Cachoeiras & 22 & 9,24 & $(5,88 ; 13,66)$ & \multirow{8}{*}{$<0,001$} \\
\hline & Aldeia Bacaiuval & 36 & 15,13 & $(10,82 ; 20,32)$ & \\
\hline & Aldeia Bacaval & 56 & 23,53 & $(18,29 ; 29,44)$ & \\
\hline & Aldeia Chapada & 20 & 8,40 & $(5,21 ; 12,68)$ & \\
\hline & Aldeia Sacre 2 & 14 & 5,88 & $(3,25 ; 9,67)$ & \\
\hline & Aldeia Seringal & 25 & 10,5 & $(6,91 ; 15,11)$ & \\
\hline & Aldeia Utiariti & 33 & 13,87 & $(9,74 ; 18,92)$ & \\
\hline & Aldeia Wazare & 32 & 13,44 & $(9,38 ; 18,45)$ & \\
\hline \multirow{4}{*}{$\begin{array}{l}\text { Atividade } \\
\text { que realiza } \\
\text { na aldeia }\end{array}$} & $\begin{array}{l}\text { Agricultura, caça, } \\
\text { pesca e coleta }\end{array}$ & 61 & 25,63 & $(20,21 ; 31,67)$ & \multirow{4}{*}{0,003} \\
\hline & $\begin{array}{l}\text { Cuidados com a casa } \\
\text { e com a família }\end{array}$ & 80 & 33,61 & $(27,64 ; 40,00)$ & \\
\hline & Estudante & 39 & 16,39 & $(11,92 ; 21,71)$ & \\
\hline & Brincadeiras & 58 & 24,37 & $(19,06 ; 30,33)$ & \\
\hline \multirow{2}{*}{$\begin{array}{l}\text { Realiza } \\
\text { atividades } \\
\text { fora da aldeia }\end{array}$} & Não & 191 & 80,25 & $(74,62 ; 85,11)$ & \multirow{2}{*}{$<\mathbf{0 , 0 0 1}$} \\
\hline & Sim & 47 & 19,75 & $(14,89 ; 25,38)$ & \\
\hline
\end{tabular}

Fonte: Autores

IC 95\%: intervalo de confiança de $95 \%$. 
Com relação aos níveis pressóricos, 31,51\% PAS e 38,34\% PAD). Ressalta-se um observou-se na Tabela 2 que tanto a PA, PAS número significativo de pessoas com hipertensão e PAD demonstram predomínio de indígenas no estágio 1, PA $(21,85 \%)$, PAS $(14,29 \%)$ e PAD com padrões pressóricos normais $(32,35 \%$ PA, $\quad(17,65 \%)(p<0,001)$.

Tabela 2 - Frequência, porcentagem e intervalo de confiança de $95 \%$ das variáveis de classificação PA, PAS e PAD de 238 indígenas do estado de Mato Grosso, Brasil, 2016.

Variáveis
Categorias
Frequência

(n)

\section{Porcentagem}

(\%)

\section{IC 95\%}

\section{Classificação PA}

Hipertensão estágio $1 \quad 52$

Hipertensão estágio 2

Hipertensão estágio 3

Limítrofe

Normal

Ótima

52

10

4

48

77

47
21,85

4,20

1,68

20,17

32,35

19,75
$(16,77 ; 27,64)$

$(2,03 ; 7,59)$

$(0,46 ; 4,25)$

$(15,26 ; 25,83)$

$(26,45 ; 38,70)$

$(14,89 ; 25,38)$

\section{Classificação PAS}

Hipertensão estágio
Hipertensão estágio 2
Hipertensão estágio
Limítrofe
Normal
Ótima

34

7

1

56

75

65
14,29

2,94

0,42

23,53

31,51

27,31
$(10,10 ; 19,39)$

$(1,19 ; 5,97)$

$(0,01 ; 2,32)$

$(18,29 ; 29,44)$

$(25,66 ; 37,83)$

$(21,76 ; 33,44)$

$<0,001$

\section{Classificação PAD}

\begin{tabular}{lcccc} 
Hipertensão estágio 1 & 42 & 17,65 & $(13,02 ; 23,10)$ & \\
Hipertensão estágio 2 & 7 & 2,94 & $(1,19 ; 5,97)$ & \\
Hipertensão estágio 3 & 3 & 1,26 & $(0,26 ; 3,64)$ & $<\mathbf{0 , 0 0 1}$ \\
Limítrofe & 13 & 5,46 & $(2,94 ; 9,16)$ & \\
Normal & 91 & 38,24 & $(27,64 ; 40,00)$ & \\
Ótima & 80 & 33,61 & $(27,64 ; 40,00)$ & \\
\hline
\end{tabular}

Fonte: Autores

IC 95\%: intervalo de confiança de $95 \%$.

$\mathrm{Na}$ análise bivariada da PA, PAS e PAD em relação aos aspectos sociodemográficos, conforme apresentado nas Tabelas 3, 4 e 5, foram identificadas significâncias estatísticas para PA e PAS nas variáveis independentes sexo masculino, idade que supera os 60 anos e realizar a atividade de agricultura, caça, pesca e coleta. Observa-se que a PAD apresentou as mesmas significâncias estatísticas supracitadas, porém sem associação com a idade. 
Tabela 3 - Associação entre Classificação PA dos indígenas com as variáveis sociodemográficas, Mato Grosso, Brasil, 2016.

\begin{tabular}{|c|c|c|c|c|c|c|c|c|}
\hline & & & Classific & :ão $\mathbf{I}$ & & & & \\
\hline Variáveis & Categoria & $\begin{array}{l}\text { Hipe } \\
123 \mathrm{e}\end{array}$ & $\begin{array}{l}\text { ensão } \\
\text { mítrofe }\end{array}$ & & $\begin{array}{l}\text { mal } \\
\text { ma }\end{array}$ & $\mathbf{R} \mathbf{P}_{\mathbf{b}}$ & IC $95 \%$ & $\mathbf{p}$ \\
\hline & & $\mathbf{N}$ & $\%$ & $\mathbf{n}$ & $\%$ & & & \\
\hline Gênero & Masculino & 71 & 60,68 & 46 & 39,32 & 1,71 & $(1,29 ; 2,26)$ & $<\mathbf{0 , 0 0 1}$ \\
\hline & Feminino & 43 & 35,54 & 78 & 64,46 & 1,00 & - & - \\
\hline Faixa etária & $<20$ anos & 27 & 41,54 & 38 & 58,46 & 1,00 & - & - \\
\hline & 20 a 59 anos & 73 & 48,03 & 79 & 51,97 & 1,16 & $(0,83 ; 1,61)$ & 0,380 \\
\hline & 60 anos ou mais & 14 & 66,67 & 7 & 33,33 & 1,60 & $(1,06 ; 2,44)$ & 0,045 \\
\hline Escolaridade & Analfabeto & 12 & 60,00 & 8 & 40,00 & 1,44 & $(0,67 ; 3,08)$ & 0,314 \\
\hline & $\begin{array}{l}\text { Ensino } \\
\text { fundamental }\end{array}$ & 67 & 50,00 & 67 & 50,00 & 1,20 & $(0,60 ; 1,39)$ & 0,580 \\
\hline & Ensino médio & 30 & 42,25 & 41 & 57,75 & 1,01 & $(0,49 ; 2,09)$ & 0,97 \\
\hline & Ensino superior & 5 & 41,67 & 7 & 58,33 & 1,00 & - & - \\
\hline Local de & Aldeia Sacre 2 & 8 & 57,14 & 6 & 42,86 & 1,40 & $(0,71 ; 2,75)$ & 0,342 \\
\hline residência & Aldeia Chapada & 11 & 55,00 & 9 & 45,00 & 1,34 & $(0,71 ; 2,55)$ & 0,361 \\
\hline & Aldeia Wazare & 17 & 53,12 & 15 & 46,88 & 1,30 & $(0,71 ; 2,36)$ & 0,377 \\
\hline & Aldeia Seringal & 13 & 52,00 & 12 & 48,00 & 1,27 & $(0,68 ; 2,38)$ & 0,447 \\
\hline & Aldeia Utiariti & 17 & 51,52 & 16 & 48,48 & 1,26 & $(0,69 ; 2,30)$ & 0,440 \\
\hline & Aldeia Bacaiuval & 16 & 44,44 & 20 & 55,56 & 1,09 & $(0,58 ; 2,02)$ & 0,792 \\
\hline & Aldeia Bacaval & 23 & 41,07 & 33 & 58,93 & 1,01 & $(0,56 ; 1,82)$ & 0,990 \\
\hline & $\begin{array}{l}\text { Aldeia } \\
4 \text { Cachoeiras }\end{array}$ & 9 & 40,91 & 13 & 59,09 & 1,00 & - & - \\
\hline $\begin{array}{l}\text { Atividade } \\
\text { que realiza } \\
\text { na aldeia }\end{array}$ & $\begin{array}{l}\text { Agricultura, } \\
\text { caça, pesca } \\
\text { e coleta }\end{array}$ & 45 & 73,77 & 16 & 26,23 & 1,69 & $(1,15 ; 2,49)$ & 0,002 \\
\hline & $\begin{array}{l}\text { Cuidados } \\
\text { com a casa } \\
\text { e com a família }\end{array}$ & 31 & 38,75 & 49 & 61,25 & 0,89 & $(0,57 ; 1,40)$ & 0,613 \\
\hline & Estudante & 21 & 36,21 & 37 & 63,79 & 0,83 & $(0,51 ; 1,36)$ & 0,465 \\
\hline & Brincadeiras & 17 & 43,59 & 22 & 56,41 & 1,00 & - & - \\
\hline Realiza & Não & 93 & 48,69 & 98 & 51,31 & 1,09 & $(0,77 ; 1,55)$ & 0,622 \\
\hline $\begin{array}{l}\text { atividades } \\
\text { fora da aldeia }\end{array}$ & Sim & 21 & 44,68 & 26 & 55,32 & 1,00 & - & - \\
\hline
\end{tabular}

Fonte: Autores

$\mathrm{RP}_{\mathrm{b}}$ : razão de prevalência bruta. IC 95\%: intervalo de confiança de $95 \%$. p: valor de p para o teste do qui-quadrado; os valores destacados em negrito são estatisticamente significantes ao nível de $5 \%$. 
Tabela 4 - Associação entre Classificação PAS dos indígenas com as variáveis sociodemográficas, Mato Grosso, Brasil, 2016.

\begin{tabular}{|c|c|c|c|c|c|c|c|c|}
\hline \multirow{3}{*}{ Variáveis } & \multirow{3}{*}{ Categoria } & \multicolumn{4}{|c|}{ Classificação PAS } & \multirow{3}{*}{$\mathbf{R P}_{\mathbf{b}}$} & \multirow{3}{*}{ IC 95\% } & \multirow{3}{*}{$\mathbf{p}$} \\
\hline & & \multicolumn{2}{|c|}{$\begin{array}{l}\text { Hipertensão } \\
123 \text { e limítrofe }\end{array}$} & \multicolumn{2}{|c|}{$\begin{array}{l}\text { Normal } \\
\text { e ótima }\end{array}$} & & & \\
\hline & & $\mathbf{N}$ & $\%$ & $\mathbf{n}$ & $\%$ & & & \\
\hline \multirow[t]{2}{*}{ Gênero } & Masculino & 63 & 53,85 & 54 & 46,15 & 1,86 & $(1,34 ; 2,58)$ & $<0,001$ \\
\hline & Feminino & 35 & 28,93 & 86 & 71,07 & 1,00 & - & - \\
\hline \multirow[t]{3}{*}{ Faixa etária } & $<20$ anos & 21 & 32,31 & 44 & 67,69 & 1,00 & - & - \\
\hline & 20 a 59 anos & 63 & 41,45 & 89 & 58,55 & 1,28 & $(0,86 ; 1,91)$ & 0,205 \\
\hline & 60 anos ou mais & 14 & 66,67 & 7 & 33,33 & 2,06 & $(1,28 ; 3,28)$ & 0,005 \\
\hline \multirow[t]{4}{*}{ Escolaridade } & Analfabeto & 12 & 60,00 & 8 & 40,00 & 2,40 & $(0,85 ; 6,81)$ & 0,055 \\
\hline & $\begin{array}{l}\text { Ensino } \\
\text { fundamental }\end{array}$ & 57 & 42,54 & 77 & 57,46 & 1,70 & $(0,63 ; 4,62)$ & 0,360 \\
\hline & Ensino médio & 26 & 36,62 & 45 & 63,38 & 1,47 & $(0,52 ; 4,09)$ & 0,528 \\
\hline & Ensino superior & 3 & 25,00 & 9 & 75,00 & 1,00 & - & - \\
\hline \multirow{8}{*}{$\begin{array}{l}\text { Local de } \\
\text { residência }\end{array}$} & Aldeia Sacre 2 & 8 & 57,14 & 6 & 42,86 & 1,40 & $(0,71 ; 2,75)$ & 0,342 \\
\hline & Aldeia Chapada & 9 & 45,00 & 11 & 55,00 & 1,10 & $(0,55 ; 2,21)$ & 0,789 \\
\hline & Aldeia Wazare & 14 & 43,75 & 18 & 56,25 & 1,07 & $(0,57 ; 2,02)$ & 0,836 \\
\hline & Aldeia Seringal & 11 & 44,00 & 14 & 56,00 & 1,08 & $(0,55 ; 2,10)$ & 0,831 \\
\hline & Aldeia Utiariti & 15 & 45,45 & 18 & 54,55 & 1,11 & $(0,59 ; 2,08)$ & 0,739 \\
\hline & Aldeia Bacaiuval & 12 & 33,33 & 24 & 66,67 & 0,82 & $(0,41 ; 1,61)$ & 0,560 \\
\hline & Aldeia Bacaval & 20 & 35,71 & 36 & 64,29 & 0,87 & $(0,47 ; 1,61)$ & 0,669 \\
\hline & $\begin{array}{l}\text { Aldeia } \\
4 \text { Cachoeiras }\end{array}$ & 9 & 40,91 & 13 & 59,09 & 1,00 & - & - \\
\hline \multirow[t]{4}{*}{$\begin{array}{l}\text { Atividade } \\
\text { que realiza } \\
\text { na aldeia }\end{array}$} & $\begin{array}{l}\text { Agricultura, } \\
\text { caça, pesca } \\
\text { e coleta }\end{array}$ & 39 & 63,93 & 22 & 36,07 & 1,56 & $(1,02 ; 2,37)$ & 0,025 \\
\hline & $\begin{array}{l}\text { Cuidados } \\
\text { com a casa } \\
\text { e com a família }\end{array}$ & 27 & 33,75 & 53 & 66,25 & 0,82 & $(0,51 ; 1,34)$ & 0,438 \\
\hline & Estudante & 16 & 27,59 & 42 & 72,41 & 0,67 & $(0,38 ; 1,18)$ & 0,167 \\
\hline & Brincadeiras & 16 & 41,03 & 23 & 58,97 & 1,00 & - & - \\
\hline \multirow{2}{*}{$\begin{array}{l}\text { Realiza } \\
\text { atividades } \\
\text { fora da aldeia }\end{array}$} & Não & 80 & 41,88 & 111 & 58,12 & 1,09 & $(0,73 ; 1,63)$ & 0,654 \\
\hline & Sim & 18 & 38,30 & 29 & 61,70 & 1,00 & - & - \\
\hline
\end{tabular}

Fonte: Autores

$\mathrm{RP}_{\mathrm{b}}$ : razão de prevalência bruta. IC 95\%: intervalo de confiança de 95\%. p: valor de p para o teste do qui-quadrado; os valores destacados em negrito são estatisticamente significantes ao nível de 5\%. 
Tabela 5 - Associação entre Classificação PAD dos indígenas com as variáveis sociodemográficas, Mato Grosso, Brasil, 2016.

\begin{tabular}{|c|c|c|c|c|c|c|c|c|}
\hline \multirow{3}{*}{ Variáveis } & \multirow{3}{*}{ Categoria } & \multicolumn{4}{|c|}{ Classificação PAD } & \multirow{3}{*}{$\mathbf{R} \mathbf{P}_{\mathrm{b}}$} & \multirow{3}{*}{ IC 95\% } & \multirow{3}{*}{$\mathbf{p}$} \\
\hline & & \multicolumn{2}{|c|}{$\begin{array}{l}\text { Hipertensão } \\
123 \text { e limítrofe }\end{array}$} & \multicolumn{2}{|c|}{$\begin{array}{l}\text { Normal } \\
\text { e ótima }\end{array}$} & & & \\
\hline & & $\mathbf{N}$ & $\%$ & $\mathbf{n}$ & $\%$ & & & \\
\hline \multirow[t]{2}{*}{ Gênero } & Masculino & 45 & 38,46 & 72 & 61,54 & 2,29 & $(1,44 ; 3,63)$ & $<0,001$ \\
\hline & Feminino & 20 & 16,81 & 99 & 83,19 & 1,00 & - & - \\
\hline \multirow[t]{3}{*}{ Faixa etária } & $<20$ anos & 18 & 27,69 & 47 & 72,31 & 1,00 & - & - \\
\hline & 20 a 59 anos & 43 & 28,67 & 107 & 71,33 & 1,04 & $(0,65 ; 1,65)$ & 0,884 \\
\hline & 60 anos ou mais & 4 & 19,05 & 17 & 80,95 & 0,69 & $(0,26 ; 1,81)$ & 0,430 \\
\hline \multirow[t]{4}{*}{ Escolaridade } & Analfabeto & 6 & 30,00 & 14 & 70,00 & 1,20 & $(0,37 ; 3,93)$ & 1,000 \\
\hline & $\begin{array}{l}\text { Ensino } \\
\text { fundamental }(\mathrm{i} / \mathrm{c})\end{array}$ & 39 & 29,32 & 94 & 70,68 & 1,17 & $(0,42 ; 3,24)$ & 1,000 \\
\hline & Ensino médio (i/c) & 17 & 24,29 & 53 & 75,71 & 0,97 & $(0,34 ; 2,81)$ & 1,000 \\
\hline & Ensino superior $(\mathrm{i} / \mathrm{c})$ & 3 & 25,00 & 9 & 75,00 & 1,00 & - & - \\
\hline \multirow{8}{*}{$\begin{array}{l}\text { Local de } \\
\text { residência }\end{array}$} & Aldeia Sacre 2 & 6 & 42,86 & 8 & 57,14 & 1,50 & $(0,61 ; 3,72)$ & 0,477 \\
\hline & Aldeia Chapada & 8 & 40,00 & 12 & 60,00 & 1,40 & $(0,59 ; 3,32)$ & 0,440 \\
\hline & Aldeia Wazare & 9 & 28,13 & 23 & 71,88 & 0,98 & $(0,41 ; 2,36)$ & 0,972 \\
\hline & Aldeia Seringal & 2 & 8,00 & 23 & 92,00 & 0,28 & $(0,06 ; 1,24)$ & 0,117 \\
\hline & Aldeia Utiariti & 10 & 30,30 & 23 & 69,70 & 1,06 & $(0,45 ; 2,49)$ & 0,892 \\
\hline & Aldeia Bacaiuval & 10 & 27,78 & 26 & 72,22 & 0,97 & $(0,41 ; 2,29)$ & 0,949 \\
\hline & Aldeia Bacaval & 14 & 25,45 & 41 & 74,55 & 0,89 & $(0,40 ; 2,01)$ & 0,783 \\
\hline & $\begin{array}{l}\text { Aldeia } \\
4 \text { Cachoeiras }\end{array}$ & 6 & 28,57 & 15 & 71,43 & 1,00 & - & - \\
\hline \multirow[t]{4}{*}{$\begin{array}{l}\text { Atividade } \\
\text { que realiza } \\
\text { na aldeia }\end{array}$} & $\begin{array}{l}\text { Agricultura, } \\
\text { caça, pesca } \\
\text { e coleta }\end{array}$ & 34 & 55,74 & 27 & 44,26 & 3,53 & $(1,64 ; 7,61)$ & $<0,001$ \\
\hline & $\begin{array}{l}\text { Cuidados } \\
\text { com a casa } \\
\text { e com a família }\end{array}$ & 12 & 15,19 & 67 & 84,81 & 0,96 & $(0,39 ; 2,37)$ & 0,933 \\
\hline & Estudante & 13 & 22,41 & 45 & 77,59 & 1,42 & $(0,59 ; 3,41)$ & 0,426 \\
\hline & Brincadeiras & 6 & 15,79 & 32 & 84,21 & 1,00 & - & - \\
\hline \multirow{2}{*}{$\begin{array}{l}\text { Realiza } \\
\text { atividades } \\
\text { fora da aldeia }\end{array}$} & Não & 51 & 26,98 & 138 & 73,02 & 0,91 & $(0,55 ; 1,49)$ & 0,70 \\
\hline & Sim & 14 & 29,79 & 33 & 70,21 & 1,00 & - & - \\
\hline
\end{tabular}

Fonte: Autores

$\mathrm{RP}_{\mathrm{b}}$ : razão de prevalência bruta. IC 95\%: intervalo de confiança de $95 \%$. p: valor de p para o teste do qui-quadrado; os valores destacados em negrito são estatisticamente significantes ao nível de 5\%. (i/c): incompleto/completo. 


\section{Discussão}

De todos os indivíduos Haliti-Paresí que participaram do estudo (50,84\%) eram do sexo feminino, achado semelhante em outros estudos com as tribos Xavante e Kaingang. ${ }^{(11-12)}$ Do total desses indígenas com idade superior a 10 anos que residem na terra indígena Utiairiti, 91,60\% são alfabetizados e superam a média nacional $(69,70 \%)$ e da Região Centro-Oeste (76,90\%), porém seguem o perfil nacional da população não indígena, ou seja, a grande maioria desses alunos $(91,90 \%)$ está cursando o ensino fundamental. ${ }^{(9)}$

Resultados semelhantes foram encontrados na pesquisa entre os Xavantes de Mato Grosso em que $61,90 \%$ com idade entre 15 e 29 anos sabiam ler e escrever e as maiores frequências de alfabetizados foram entre os homens. ${ }^{(13)}$ A baixa escolaridade pode ser um dos fatores de risco para o desenvolvimento das doenças crônicas devido à desigualdade de acesso aos serviços de atenção primária que consigam trabalhar os aspectos de prevenção dessas doenças. ${ }^{(14)}$ Em estudo realizado com indígenas da etnia Xerente de Tocantins houve diagnóstico de hipertensão arterial, com destaque para relação para o baixo grau de escolaridade, já que quase metade eram analfabetos e o restante não havia concluído o ensino fundamental. ${ }^{(15-16)}$

No cenário brasileiro e nas comunidades indígenas é notável a primazia feminina. Mesmo diante a tantas evoluções ao longo dos anos e à conquista do espaço no mercado de trabalho, as mulheres ainda são responsáveis por executar as tarefas domésticas. Historicamente os homens desempenham de forma predominante o papel de provedor da família, e as mulheres são responsáveis pelo cuidado do lar, atividade esta que funciona como contrapartida dado ao sustento financeiro do marido. ${ }^{(17)}$ Contudo, nas comunidades indígenas a mulher possui responsabilidades importantes em relação às atividades desenvolvidas. De modo geral a divisão do trabalho nos grupos indígenas do Brasil se dá pela divisão por sexo e idade. Isto é, um homem faz o que todos os outros fazem, assim como as mulheres. ${ }^{(18-19)}$
Entre os Haliti-Paresí, a principal atividade por eles desempenhada são os cuidados com a casa e com a família $(33,61 \%)$, fato este que pode ser explicado devido a maior parte de esses indígenas serem mulheres e esta ser uma atividade relacionada ao sexo feminino. Porém, no cotidiano dos Haliti-Paresí a maioria das atividades para ambos os sexos é desempenhada na própria aldeia. Aos homens cabe o trabalho nas lavouras, plantão para recebimento do pedágio na estrada que passa pelas terras indígenas, além da caça, pesca e de trabalhos gerenciais na associação e na Funai. As mulheres se dedicam ao artesanato, ao cuidado com as casas e crianças. Já a maioria das pessoas idosas recebe aposentadoria, mas mantém o trabalho nas roças domésticas e realiza também as coletas de frutas e sementes, além da caça e pesca. ${ }^{(10)}$

Sabe-se que a etiologia da HAS é multifatorial, porém estudos apontam que alguns fatores podem estar associados ao aumento dos níveis pressóricos como ruídos, trabalho em turnos, exposição a agentes químicos e estresse. ${ }^{(20)}$ Estudo nacional aponta para o conceito de que os trabalhadores que desempenham trabalho braçal sofrem maiores alterações de níveis pressóricos, ou seja, onde há maior presença de esforço físico. ${ }^{(21)}$ Entre as populações indígenas não foram encontrados relatos de alteração dos níveis pressóricos relacionados às atividades de caça, pesca, coleta e agricultura; entretanto, autores destacam uma maior associação de HAS com obesidade, tabagismo e consumo de bebidas alcoólicas. ${ }^{(4,22)}$

Nas décadas de 70 e 80 os estudos sobre hipertensão em populações indígenas brasileiras eram praticamente inexistentes; essas populações vivenciam amplas modificações em seus hábitos socioculturais, econômicos e comportamentais que impactam diretamente na prevalência das doenças crônicas como a hipertensão. ${ }^{(23)} \mathrm{A}$ obesidade e fatores comportamentais estão na base desse problema, que associados à transição nutricional vivenciada pelos indígenas brasileiros contribuíram para o desenvolvimento da hipertensão arterial. ${ }^{(24)}$

O perfil nutricional é um dos principais fatores que podem condicionar a hipertensão 
arterial. Um estudo sobre o perfil nutricional dos indígenas revelou que $27,60 \%$ dos indígenas estavam com sobrepeso e $5,20 \%$ com obesidade, associados ao fato dessa comunidade ter migrado de Alagoas para Minas Gerais há 19 anos, alterando seus hábitos alimentares. ${ }^{(25)} \mathrm{A}$ migração para territórios diferentes dos nativos pode ser um fator importante no desenvolvimento de doenças crônicas, podendo ser evidenciado em outro estudo envolvendo três etnias que migraram para o Alto Xingu (MT), em que a análise do perfil metabólico detectou que todos apresentaram semelhantes fatores de risco para doenças cardiovasculares, dentre estes, os Aruak com 51,80\% de sobrepeso, seguido por $15 \%$ de obesidade, além do destaque para presença de $77,10 \%$ de dislipidemia e $37,70 \%$ com níveis pressóricos elevados. ${ }^{(18)}$

As descrições sobre níveis tensionais com indígenas Suruí de Rondônia, Kaingang da Terra Indígena Xapecó (SC), Xukuru do Ororuba (PE), Aldeia Jaguapiru (MS) apresentaram um aumento na prevalência de hipertensão arterial. ${ }^{(6-8,26)}$ Há heterogeneidade por regiões brasileiras para hipertensão, ou seja, menor prevalência na Região Norte $(3,60 \%)$ e cinco vezes maior nas Regiões Sul, Sudeste e Centro-Oeste. ${ }^{(27)}$

Ao avaliar indígenas da tribo Khisêdjê de ambos os sexos, com idade $\geq 20$ anos, $40 \%$ eram do sexo feminino, e em ambos os sexos observouse maior porcentagem de indígenas na faixa etária de 20 a 29 anos $(38,20 \%)$ e prevalência de hipertensão arterial de 6,80\%. ${ }^{(18)}$ Em análise dos níveis pressóricos dos índios Xavantes de Mato Grosso, em ambos os sexos foi observado que estavam abaixo dos valores médios observados na população brasileira adulta em geral (20\%), ou seja, a prevalência foi de $17,20 \%$ nos indígenas e não houve diferença de sexo.(11) Ao contrário do estudo anterior, entre os Haliti-Paresí houve diferença estatisticamente significante em relação ao sexo, pois os homens apresentaram as maiores prevalências de alteração dos níveis pressóricos $(60,68 \%)$ em comparação às mulheres $(35,54 \%)$.

A condição de saúde observada entre os Haliti-Paresí do sexo masculino é uma realidade do cenário nacional. Os homens apresentam a pressão arterial menos controlada do que as mulheres, fato relacionado à dificuldade deles em reconhecer suas necessidades de saúde, cultivando o pensamento que rejeita a possibilidade de adoecer - características que dificultam a adesão ao tratamento médico e as práticas de autocuidado. ${ }^{(28)}$ Com relação às medidas da pressão arterial, $53,85 \%$ dos homens Haliti-Paresí tiveram alteração da pressão sistólica (PAS) e 38,46\% da pressão diastólica (PAD).

Entre os Kaingang ${ }^{(6)}$ os níveis médios de PAS nos homens foram maiores $(133,20 \mathrm{mmHg})$, com destaque para aqueles que estudaram 12 anos ou mais, e a média da PAD foi de $86,20 \mathrm{mmHg}$. Outras pesquisas investigaram a prevalência de níveis tensionais sugestivos de HAS em indígenas adultos e encontraram menores prevalências e menores valores médios de PAS e PAD. ${ }^{(8,29)}$ Já entre os Suruí foram registradas prevalências de HAS de 2,40\% entre os homens e 3,10\% entre as mulheres e aumento dos valores médios de PAS e PAD associados a excesso de peso. ${ }^{(8)}$

Uma associação entre PAS e PAD foi descrita apenas entre as mulheres indígenas Kaingang, diferentemente do que foi encontrado entre os Haliti-Paresí. ${ }^{(6)}$ Ainda assim, os valores médios de PAS e PAD em relação ao gênero se apresentaram um pouco distintos em cada estudo. ${ }^{(8,19)}$ No que se refere à PAS e PAD, a primeira esteve associada com a escolaridade também no sexo masculino. ${ }^{(6)}$ O grau de escolaridade entre os Haliti-Paresí não apresentou associação estatística com alterações na pressão arterial.

Em uma análise com 273 idosos foi descrito que $64,80 \%$ estavam na faixa etária de 60 a 69 anos e a prevalência da HAS foi de $67,40 \%$, sendo $68 \%$ no sexo masculino e $66,90 \%$ no sexo feminino. Essas taxas foram similares entre os Haliti-Paresí, já que $66,67 \%$ dos que possuem 60 anos ou mais registraram índices elevados de alteração na PA, fato este que pode ser explicado em decorrência dos fatores fisiológicos relacionados ao processo de envelhecimento, associado às alterações nutricionais e adoção dos maus hábitos de vida. 
As populações indígenas são consideradas como um grupo de indivíduos com a menor expectativa de vida e estado de saúde mais pobre em comparação com os não indígenas. ${ }^{(3)}$ Apesar de todos os conhecimentos e evidências da importância do diagnóstico precoce e tratamento da hipertensão arterial, controlar a doença ainda é um grande desafio para os profissionais da saúde. Vale ressaltar que as práticas preventivas não são rotina da maioria da população masculina, sendo esses cuidados predominantemente femininos. A incidência de alteração dos níveis pressóricos em indígenas, especialmente do sexo masculino, na faixa etária dos 60 anos, só reforça a importância da adoção de medidas educativas e assistências a esse grupo de risco.

Dar-se então a importância da assistência de um profissional capacitado que realize ações de promoção, prevenção e elaboração de planos estratégicos que visam reforçar sobre a adoção de hábitos saudáveis é de extrema importância para o cuidado e melhoria na qualidade de vida desses indivíduos.

\section{Conclusão}

O perfil sociodemográfico dos Haliti-Paresí demonstra uma população com predomínio de jovens, com ensino fundamental incompleto, residentes na aldeia Bacaval, que realizam em seu cotidiano as atividades de acordo com a divisão social e a minoria realiza alguma atividade fora da aldeia. A análise da PA, PAS e PAD demonstrou predomínio de indígenas com padrões pressóricos normais. Cabe ressaltar o número significativo de pessoas com normal/ótima ou limítrofe $+1+2+3$, ou seja, PA $(21,85 \%)$, PAS (14,29\%) e PAD $(17,65 \%)(p<0,001)$. Na análise bivariada foram identificadas significâncias estatísticas para PA e PAS nas variáveis independentes sexo masculino, idade que supera os 60 anos e realizar a atividade de agricultura, caça, pesca e coleta. No entanto, a PAD apresentou as mesmas significâncias estatísticas supracitadas, porém sem associação com a idade.
Os achados do estudo podem ser resultantes das mudanças de hábitos culturais, econômicos e de estilo de vida, decorrentes da transição epidemiológica que estão vivenciando, principalmente pela interação do indígena com outras sociedades.

Este estudo apresenta uma contribuição adicional para o conhecimento sobre a hipertensão arterial entre os indígenas brasileiros, pois insere associações importantes sobre a doenças e os aspectos sociodemográficos, sendo assim importante para direcionar as ações de prevenção da HA e promoção da saúde. Como limitação do estudo pode-se apontar a transversalidade e impossibilidade de se afirmar a temporalidade dos fatores associados encontrados.

Torna-se fundamental refletir sobre a adoção de políticas públicas com foco na vigilância, controle e prevenção dos fatores de risco para hipertensão, com destaque para as ações do profissional de saúde na atenção à saúde indígena, compreendendo o universo cultural dos índios para promover intervenções eficazes que possam impactar na melhoria da qualidade de vida dessas populações.

\section{Financiamento}

Fundação de Amparo à Pesquisa do Estado de Mato Grosso FAPEMAT, edital Universal FAPEMAT $n^{\circ} 005 \backslash 2015$, processo $n^{\circ} 219718 \backslash 2015$.

\section{Agradecimentos}

Os autores agradecem a comunidade HalitiParesí, em especial as nove aldeias da terra indígena Utiariti. 


\section{Referências}

1 Sociedade Brasileira de Cardiologia. $7^{\text {a }}$ diretriz brasileira de hipertensão arterial. Arq Bras Cardiol. [Internet] 2016 [citado 2019 jan 2]; 107(supl 3):1-12. Disponível em: http://publicacoes.cardiol.br/2014/ diretrizes/2016/05_HIPERTENSAO_ ARTERIAL.pdf

2 Ministério da Saúde (BR). Vitigel Brasil 2016 [Internet]. 2017 [citado 2019 fev 5]. Disponível em: https://portalarquivos2.saude.gov.br/images/ pdf/2018/marco/02/vigitel-brasil-2016.pdf

3 Oliveira GF, Oliveira TR, Ikejiri AT, Andraus MP, Glavão TF, Silva MT, et al. Prevalence of hypertension and associated factors in an indigenous community of central Brazil: a population-based study. PLos One. 2015;9(1):302-10. doi: 10.1371/journal. pone. 0086278 .

4 Ferreira AA, Souza Filho ZA, Gonçalves MJF, Santos J, Pierin AMG. Relationship between alcohol drinking and arterial hypertension in indigenous people of the Mura ethnics. Brazil. PloS One. 2017;12(8):1-17. doi: 10.1371/ journal.pone.0182352.

5 Crepaldi GB. Alimentação indígena em Mato Grosso: educação ambiental e sustentabilidade entre etnias de estudantes da faculdade indígena intercultural [dissertação] [Internet]. Cáceres: Universidade do Estado de Mato Grosso; 2012 [citado 2019 mar 8]. Disponível em: http:// portal.unemat.br/media/oldfiles/ppgca/docs/ gabrielle_crepaldi.pdf

6 Bresan D, Bastos LJ, Leite MS. Epidemiology of high blood pressure among the Kaingang people on the Xapecó Indigenous Land in Santa Catarina State, Brazil, 2013. Cad Saúde Pública. 2015;31(2):331-44. doi: 10.1590/0102$311 X 00058714$.

7 Oliveira GF. Prevalência de fatores de risco cardiometabólicos em comunidade indígena no brasil central: um estudo transversal de base populacional [tese] [Internet]. Brasília: Universidade de Brasília; 2014 [citado 2019 jan 5]. Disponível em: http://repositorio.unb.br/ handle/10482/17247
8 Tavares FG, Coimbra Junior CEA, Carlos EA, Cardoso AM. Níveis tensionais de adultos indígenas Suruí, Rondônia, Brasil. Ciênc Saúde Coletiva. 2013;18(5):1399-409. doi: 10.1590/S1413-81232013000500025.

9 Terças ACP, Nascimento VF, Hattori TY, Zenazokenae LE, Atanaka M, Lemos, ERS. Os Haliti-Paresí: uma reflexão sobre saúde e demografia da população residente nas terras indígenas Haliti-Paresí. Espaço Ameríndio. 2016;10(1):226-53. doi: 10.22456/19826524.60301 .

10 Canova L. Doces bárbaros: imagens dos índios Paresi no contexto da conquista portuguesa em Mato Grosso (1719-1757) [Dissertação] [Internet]. Cuiabá: Universidade Federal de Mato Grosso; 2001. [citado 2019 jan 5]. Disponível em: http://livros01.livrosgratis. com.br/cp000214.pdf

11 Dal Fabbro AL, Franco LJ, Silva AS, Sartorelli DS, Soares LP, Franco LF, et al. High prevalence of type 2 diabetes mellitus in xavante indians from mato grosso, brazil. Ethn Dis. 2014;24(1):35-40.

12 Borghi AC, Carreira L. Condições de vida e saúde do idoso indígena Kaingang. Esc Anna Nery. 2015;19(3):211-517. doi: 10.5935/1414-8145.20150068.

13 Souza LG, Gugelmin SS, Cunha BCB, Atanaka M. Os indígenas Xavante no censo demográfico de 2010. Rev. Bras. Estud. Popul. 2016;33(2):327-47. doi: 10.20947/s0102$30982016 \mathrm{a} 0025$.

14 Arias JAC. Prevalencia de factores de riesgo cardiovascularenindígenas deRiosucio-Caldas, 2010-2011. Rev Med UPB. [Internet] 2012 [citado 2019 jan 5];31(2):113-26. Disponível em: https://revistas.upb.edu.co/index.php/ Medicina/article/viewFile/1759/1696

15 Rodrigues KN, Santos, NSS. Percepção do Indígena Xerente sobre a Hipertensão Arterial Sistêmica no Tocantins. Rev. Pesqui. Cuid. Fundam. 2016;8(2):4549-4562. doi: 10.9789/2175-5361.2016.v8i2.4549-4562. 
16 Santos KMP, Silva RJN. O uso dos recursos naturais do cerrado para produção artesanal: um estudo de caso entre os índios krahô. Nera. 2016;19(33):30-46.

17 Sousa LP, Guedes DR. A desigual divisão sexual do trabalho: um olhar sobre a última década. Estud. Av. 2016;30(87):123-39. doi: 10.1590/S0103-40142016.30870008.

18 Santos KM, Tsutsui MLS, Galvao PPO, Mazzucchetti L, Rodrigues D, Gimeno SGA. Grau de atividade física e síndrome metabólica: um estudo transversal com indígenas Khisêdjê do Parque Indígena do Xingu, Brasil. Cad. Saúde Pública. 2012;28(12):2327-38. doi: 10.1590/S0102-311X2012001400011.

19 Oliveira GF, Oliveira TR, Rodrigues FF, Correa LF, Arruda TB, Casulari LA. Prevalence of metabolic syndrome in the indigenous population, aged 19 to 69 years, from Jaguapiru Village, Dourados (MS), Brazil. Ethn Dis. 2011;21(3):301-6.

20 Andrade RCV, Fernandes RCP. Hipertensão arterial e trabalho: fatores de risco. Rev Bras Med Trab. 2016;14(3):252-61. doi: 10.5327/ Z1679-443520164015.

21 Aleixo LMSB. Prevalência e fatores associados à hipertensão arterial sistêmica (HAS) autorreferida entre trabalhadores do serviço público municipal de Goiânia [Dissertação] [Internet]. Goiânia (GO): Universidade Federal de Goiás; 2015. Disponível em: https:// repositorio.bc.ufg.br/tede/handle/tede/6330

22 Chagas CA. Prevalência estimada e fatores associados à hipertensão arterial em indígenas Krenak do estado de Minas Gerais [Dissertação]. Belo Horizonte (MG): Universidade Federal de Minas Gerais; 2018. Disponível em: http://www.enf.ufmg.br/ images/enfermagem/Cristiane_Alvarenga_ Chagas_2018.pdf

23 Souza Filho ZA, Ferreira AA, Santos BS, Pierin AMG. Hypertension prevalence among indigenous populations in Brazil: a systematic review with meta-analysis. Rev. Esc. Enferm. USP. 2015;49(6):012-22. doi: 10.1590/S0080623420150000600019 .
24 Favaro TR, Santos RV, Cunha GM, Leite IDAC, Coimbra Junior CEA. Obesity and overweight in adult Xukuru of Ororubá Indians, Pernambuco State, Brazil: magnitude and associated socioeconomic and demographic factors. Cad. Saúde Pública. 2015;31(8):168597. doi: 10.1590/0102-311X00086014.

25 Simoes BS, Machado Coelho GLL, Pena JL, Freitas SN. Nutritional profile of the Xukuru-Kariri indigenous people in the state of Minas Gerais in accordance with different anthropometric and body composition indicators. Cien Saude Colet. 2013;8(2):40511. doi: 10.1590/S1413-81232013000200012.

26 Barbosa JMV. Prevalência e fatores associados à pressão arterial elevada no povo Indígena Xukuru do Ororubá, Pesqueira-PE [Dissertação] [Internet]. Recife (PE): Fundação Oswaldo Cruz; 2013. [citado 2019 jan 5]. Disponível em: http://bdtd.ibict.br/vufind/Record/CRUZ c5bf5cfbfadadee $11 \mathrm{ff} 67 \mathrm{c} 233 \mathrm{c} 8 \mathrm{fc} 3 \mathrm{f0}$

27 Coimbra CEA Jr, Santos RV, Welch JR, Cardoso AM, Souza MC, Garnelo L, et al. The first national survey of indigenous people's health and nutrition in brazil: rationale, methodology, and overview of results. BMC Public Health. 2013;13(52):1471-2458. doi: 10.1186/14712458-13-52.

28 Portela PP, Mussi FC, Gama GGG, Santos CAST. Fatores associados ao descontrole da pressão arterial em homens. Acta Paul. Enferm. 2016;29(3):307-15. doi: 10.1590/19820194201600043 .

29 Meyerfreund D, Gonçalves C, Cunha R, Pereira AC, Krieger JE, Mill JG. Agedependent increase in blood pressure in two different Native American communities in Brazil. J Hypertens. 2009;27(9):1753-60. doi: 10.1097/HJH.0b013e32832e0b2b.

30 Esperandio EM, Espinosa MM, Martins MAS, Guimarães LV, Lopez MAL, Scala LCN. Prevalência e fatores associados à hipertensão arterial em idosos de municípios da Amazônia Legal, MT. Rev. Bras. Geriatr. Gerontol. 2013;16(3):481-93. doi: 10.1590/S180998232013000300007 . 
Recebido em: 18 abr. 2019 Aceito em: 7 fev. 2020 
Moura, A. C. P. et al. 